\title{
COVID-19 : Un argument en faveur de la collecte de données sur les populations racialisées au Canada et à l'étranger
}

\author{
Emily Thompson ${ }^{1}$, Rojiemiahd Edjoc ${ }^{1 *}$, Nicole Atchessi', Megan Striha ${ }^{1}$, Imran Gabrani-Juma1', \\ Thomas Dawson ${ }^{1}$
}

\section{Résumé}

Les populations racialisées présentent invariablement des résultats en matière de santé plus faible, et ce, partout dans le monde. Cette tendance est devenue encore plus évidente dans le sillage de la pandémie de la maladie à coronavirus 2019 (COVID-19). Dans les pays où des données désagrégées selon la race sont régulièrement recueillies, comme les États-Unis et le Royaume-Uni, des rapports préliminaires ont révélé que les populations racialisées courent un risque accru d'infection et de mortalité liées à la COVID-19. Des schémas semblables se dessinent au Canada, mais, en l'absence de données individuelles, ils reposent sur des mesures indirectes comme la diversité des quartiers pour tenir compte de la race. II s'ensuit que la collecte de données désagrégées selon la race au Canada est un élément crucial pour identifier les personnes susceptibles d'obtenir de faibles résultats liés à la COVID-19 et élaborer des interventions ciblées en santé publique afin d'atténuer les risques parmi les populations racialisées du Canada. Compte tenu de cet écart persistant, il est de la plus haute importance de préconiser un accès rapide à ces données en raison des défis que la pandémie de COVID-19 a mis en lumière parmi les populations racialisées au Canada et dans le monde.
Cette oeuvre est mise à la disposition selon les termes de la licence internationale Creative Commons Attribution 4.0

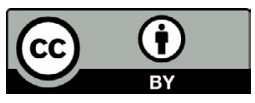

Affiliation

${ }^{1}$ Direction générale de l'infrastructure de sûreté sanitaire, Agence de la santé publique du Canada, Ottawa, ON

*Correspondance :

rojiemiahd.edjoc@canada.ca

Citation proposée : Thompson E, Edjoc R, Atchessi N, Striha M, Gabrani-Juma I, Dawson T. COVID-19 : Un argument en faveur de la collecte de données sur les populations racialisées au Canada et à l'étranger. Relevé des maladies transmissibles au Canada 2021;47(7/8):330-5. https://doi.org/10.14745/ccdr.v47i78a02f Mots-clés : COVID-19, coronavirus, race, population racialisée, minorité visible, maladies infectieuses

\section{Introduction}

La maladie à coronavirus 2019 (COVID-19) est une maladie infectieuse causée par le coronavirus du syndrome respiratoire aigu sévère 2 (SRAS-CoV-2), identifié pour la première fois en décembre 2019. Le 11 mars 2020, I'Organisation mondiale de la Santé (OMS) qualifiait la COVID-19 de pandémie. En date du 23 mars 2021, il y avait eu plus de 124 millions de cas et 2,7 millions de décès dans le monde, avec 947024 cas et 22712 décès rien qu'au Canada (1).

Des études ont cerné plusieurs facteurs associés aux taux d'infection élevés et aux résultats plus graves relatifs à la COVID-19, notamment des antécédents de maladies chroniques, le fait d'être immunodéprimé et un âge avancé $(2,3)$. Cependant, peu d'études ont exploré l'incidence de la race sur les résultats en matière de santé de la COVID-19. Dans les ouvrages scientifiques, une variété de termes sont utilisés pour décrire les populations et les personnes racialisées, et démêler ces termes et définitions nécessitera des recherches plus approfondies. Aux fins du présent article, le terme " race » réfère à la construction sociale selon laquelle les personnes sont classées en fonction de différences physiques perçues $(4,5)$. D'autres termes, comme
" minorité visible », "Noir, Asiatique et minorité ethnique » et " diversité ethnique », sont utilisés lorsque l'auteur de l'étude en question a utilisé de tels termes. Nous notons également que le terme " race " ne comprend pas les personnes autochtones, car ces collectivités sont confrontées à des inégalités distinctes en matière de santé enracinées dans un colonialisme de longue date, et l'auto-identification de l'identité autochtone devrait être distincte par rapport à la façon dont ces personnes sont racialisées par la société (4).

Il est impossible de discuter des disparités raciales en matière de santé sans d'abord aborder les déterminants sociaux des inégalités systémiques et relatives à la santé qui touchent directement les populations racialisées. Des études ont montré que des facteurs tels que le revenu, l'emploi, l'éducation et le logement diffèrent considérablement entre les groupes racialisés et non racialisés (6). Les personnes racialisées, en particulier, sont plus susceptibles d'occuper des emplois mal rémunérés offrant un accès limité aux congés de maladie payés et de vivre dans des logements mal entretenus, instables ou surpeuplés (7), des facteurs qui ont tous été associés à de faibles résultats 
relatifs à la COVID-19. Bien que ces problèmes aient été mis au premier plan à cause de la pandémie, il est important de reconnaître qu'ils sont intrinsèquement enracinés dans le racisme systémique. Le racisme systémique est le système par lequel les politiques et les structures existantes renforcent et perpétuent les inégalités raciales (8), lequel est invariablement lié à de faibles résultats en matière de santé générale et physique (9-11). Un commentaire initial de Yancy souligne à quel point les interventions proposées dans le cadre de la COVID-19, comme la distanciation physique, le télétravail et l'acceptation d'un congé, sont des questions de privilèges et pourraient ne pas être accessibles aux membres des communautés marginalisées (12). Ces inégalités ne sont pas le fruit de la pandémie de COVID en soi, mais plutôt l'indication d'un problème beaucoup plus répandu de racisme intégré dans les politiques, les pratiques et les institutions sociétales. Bien que nous ne soyons pas en mesure de donner un aperçu plus détaillé du racisme systémique dans cet article, il est important de reconnaître que son incidence sur les soins de santé est considérable.

II s'ensuit que, dans la foulée de la COVID-19, il est essentiel d'obtenir des données désagrégées pour comprendre l'incidence de la pandémie sur les diverses populations du Canada. II est de plus en plus évident que les personnes racialisées sont touchées de façon disproportionnée par la pandémie; de nouvelles études aux États-Unis et au Royaume-Uni ont démontré une tendance alarmante par rapport au fardeau de la COVID-19 au sein de ces groupes, notamment des taux d'infection et de mortalité plus élevés et des conséquences plus graves liées à la maladie (13-15). Au Canada, les études explorant le lien entre les populations racialisées et la COVID-19 sont limitées et reposent actuellement sur des mesures indirectes comme la diversité des quartiers plutôt que sur des données individuelles (16). Le présent article a donc deux objectifs. Premièrement, il explore l'effet de la pandémie de COVID-19 en exposant les inégalités déjà existantes en matière de santé parmi les populations racialisées par rapport aux populations non racialisées en examinant des mesures en aval comme les taux d'infection et de mortalité liées à la COVID-19. Deuxièmement, il préconise l'amélioration de la collecte de données sur la race et l'accès rapide à ces ensembles de données afin de mieux appuyer la prise de décisions concernant les populations racialisées au Canada.

\section{Populations racialisées et incidence de la COVID-19}

Le risque accru d'infection à la COVID-19 a souvent été lié à des facteurs socioéconomiques comme un logement inapproprié et un emploi précaire $(10,17)$. La combinaison de facteurs tels que la race, le statut socioéconomique et la santé revêt une importance particulière pour les personnes racialisées, qui font régulièrement l'objet de taux plus élevés de pauvreté au travail, de logements inférieurs aux normes et de revenus plus faibles (18). Les rapports préliminaires de Santé publique Ontario publiés au cours des premiers mois de la pandémie de COVID-19 ont indiqué que les personnes vivant dans des quartiers ethniquement diversifiés ont connu des taux plus élevés de maladie, d'hospitalisation et de décès liés au COVID-19 comparativement aux personnes provenant de quartiers moins diversifiés (19). De même, I'Institute for Clinical Evaluative Sciences a publié un rapport détaillant les modèles d'analyse en laboratoire de la COVID-19 en Ontario au cours des trois premiers mois de la pandémie. Ces premières données suggèrent que les Ontariens vivant dans les collectivités ayant les proportions les plus élevées de membres de minorités visibles ou d'immigrants récents étaient trois fois plus susceptibles d'obtenir un test positif à la COVID-19 que les Ontariens vivant dans des quartiers moins diversifiés : les collectivités diversifiées indiquaient 10 cas de COVID-19 pour 100 personnes testées comparativement à 3,2 cas positifs pour 100 personnes testées dans les quartiers moins diversifiés (20). Des tendances semblables se sont manifestées aux États-Unis, où les quartiers majoritairement noirs sont associés à une augmentation des tests positifs à la COVID comparativement aux quartiers blancs (21).

Les populations racialisées ont également été citées comme étant plus susceptibles d'être des travailleurs de services essentiels, ce qui les expose à un risque plus élevé de contracter la COVID-19 en raison d'une exposition accrue aux personnes infectées (22). Au Canada, les minorités visibles représentent environ un tiers des aides-infirmiers, des aides-soignants et des préposés aux bénéficiaires, avec une proportion plus élevée de travailleurs noirs, philippins et sud-asiatiques qui exercent ces professions (23). Ce fardeau de la main-d'œuvre se dessine également aux États-Unis et au Royaume-Uni, où les populations racialisées sont plus susceptibles d'occuper des emplois de services mal rémunérés offrant un accès inadéquat aux congés de maladie payés $(14,24,25)$. Enfin, ces facteurs pourraient également avoir des conséquences biologiques importantes sur la sensibilité des individus à l'infection. On a observé qu'une exposition constante à des facteurs de stress chroniques, comme de mauvaises conditions de vie et de travail, active l'axe hypothalamo-hypophysaire, entraînant ainsi une plus grande sécrétion d'hormones de stress. On a émis l'hypothèse selon laquelle l'exposition à long terme et l'incapacité à réguler ces hormones seraient des facteurs contributifs aux maladies chroniques $(26,27)$. Pour les populations racialisées, lesquelles sont déjà confrontées à des différences importantes liées aux principaux déterminants de la santé ainsi qu'à des taux de comorbidité plus élevés, le fardeau accru engendré par la pandémie de COVID-19 pourrait entraîner un plus grand nombre d'expositions et une sensibilité accrue à la COVID-19. 


\section{Populations racialisées et mortalité attribuable à la COVID-19}

Bien que les chercheurs du Canada n'aient pas facilement accès aux données individuelles selon la race, des mesures indirectes comme la diversité des quartiers ont été utilisées pour étudier les disparités associées aux décès attribuables à la COVID-19 entre les populations racialisées et non racialisées. Une étude récente de Statistique Canada reliant les données provisoires sur la mortalité en 2020 et les données du Recensement de 2016 a montré que les taux de mortalité attribuables à la COVID-19 étaient environ deux fois plus élevés dans les quartiers canadiens où la proportion de membres des minorités visibles était plus élevée que dans ceux où la proportion était plus faible. Ce contraste était particulièrement marqué en Ontario, au Québec et en Colombie-Britannique, où les taux de mortalité étaient entre 3 et 10 fois plus élevés dans des quartiers plus diversifiés (28). De même, un premier rapport de Santé publique Ontario indiquait que les taux de mortalité étaient trois fois plus élevés dans les quartiers plus diversifiés que dans les quartiers moins diversifiés (19). Toutefois, sans accès aux données individuelles, il est difficile de déterminer les groupes qui ont le taux de mortalité le plus élevé et les raisons associées.

Au Royaume-Uni, la race a été établie comme un prédicteur important de mortalité attribuable à la COVID-19 (29).

OpenSAFELY, I'une des plus importantes études de cohorte en cours sur les décès liés à la COVID-19, a indiqué que le risque de décès liés à la COVID-19 dans les groupes ethniques minoritaires noirs et asiatiques était près de 1,5 fois plus élevé que celui des personnes blanches, et ce, même après un ajustement en fonction de l'âge, du sexe, de la privation et des comorbidités pertinentes (13). Aux États-Unis, bien qu'ils constituent moins d'un tiers de la population, les Noirs représentent 40 à $70 \%$ des décès liés à la COVID-19 dans plusieurs villes, avec des taux de mortalité presque six fois plus élevés dans les comtés principalement noirs que dans les comtés majoritairement blancs (12).

Il convient de noter que, bien que plusieurs études aient signalé un taux de mortalité plus élevé parmi les populations racialisées, le lien entre la mortalité attribuable à la COVID-19 et la race demeure flou. Un examen du Groupe des sciences émergentes de l'Agence de la santé publique du Canada a révélé une hétérogénéité considérable parmi les études portant sur la mortalité attribuable à la COVID-19 et la race, sans augmentation significative du risque de mortalité chez les Noirs ou les Asiatiques (30). De plus, des recherches plus approfondies sont nécessaires pour comprendre le lien entre la morbidité liée à la COVID-19 et la race, car les répercussions à long terme de la maladie font encore l'objet de recherches.

\section{Recommandations}

La compréhension du lien entre les répercussions de la COVID-19 et les populations racialisées est une priorité de recherche au Canada. La pandémie a souligné les inégalités de longue date des populations racialisées du Canada, mettant en évidence la nécessité de disposer de données sur la santé désagrégées selon la race. La disponibilité limitée de données canadiennes a fait en sorte que peu d'études ont été publiées sur les différences raciales dans les résultats et la mortalité liés à la COVID-19 (figure 1). Des mesures indirectes, comme la diversité des quartiers, ont permis de mieux comprendre les tendances associées à la COVID-19 entre les minorités visibles et les non-minorités (28). Cependant, une étude plus approfondie est nécessaire pour démonter les variations des résultats liés à la COVID-19 chez les différentes populations racialisées.

Figure 1: Nombre d'études d'observation sur le lien entre la race et le risque d'infection à la COVID-19, la gravité associée à celle-ci ou la mortalité attribuable à la COVID, par pays ${ }^{\mathrm{a}}$, publiées entre le $1^{\text {er }}$ juin et le 7 septembre 2020

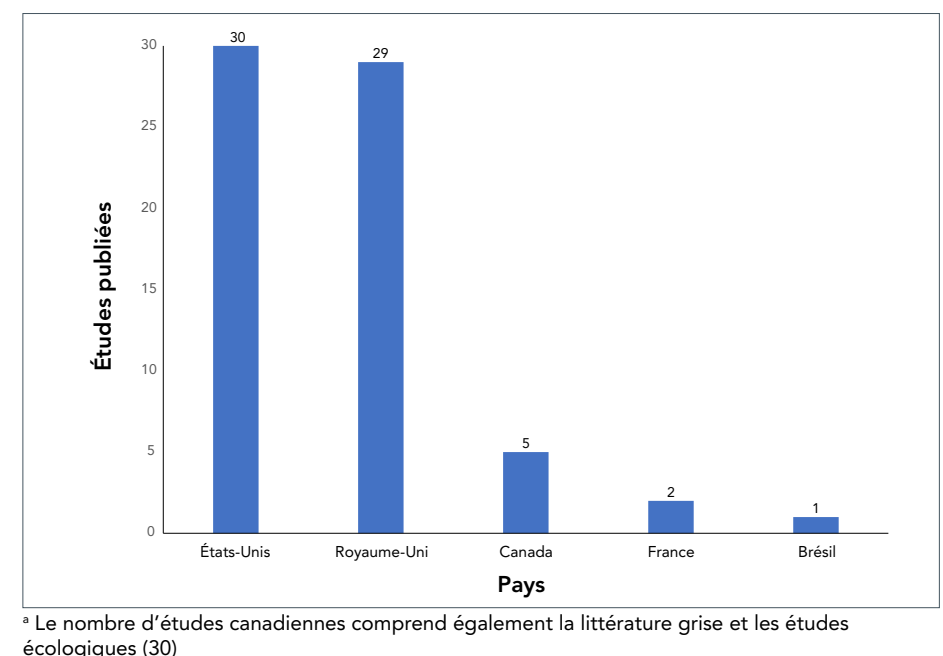

Nous reconnaissons la pression et le fardeau qu'exerce la COVID-19 sur les ressources et le personnel locaux, provinciaux et nationaux, ainsi que la difficulté de définir des variables cohérentes à tous les niveaux de gouvernement. Toutefois, il est fortement recommandé que des efforts continus soient déployés pour peaufiner la procédure de collecte de données individuelles selon la race afin d'offrir aux chercheurs un accès opportun à ces renseignements et ainsi les aider à élaborer des politiques et des mesures d'intervention en matière de santé publique. Au fur et à mesure que la pandémie progresse au Canada, des provinces comme le Manitoba et l'Ontario, ainsi que leurs autorités régionales de la santé respectives, ont indiqué leur intention d'effectuer une collecte de données désagrégées selon la race. D'autres organismes, comme l'Institut canadien 
d'information sur la santé, ont publié des normes proposées pour la collecte de données sur l'identité autochtone et selon la race, bien que d'autres données soient nécessaires pour la mise en œuvre de ce projet (4). Nous reconnaissons également l'existence d'obstacles importants à la collecte de données selon la race dans ces cas, comme un fardeau accru pour les travailleurs de la santé (31) des préoccupations en matière de vie privée (32) et la qualité et l'utilité des données (32,33). La collaboration entre les organismes de santé provinciaux et fédéraux est essentielle pour appuyer les initiatives qui visent à recueillir des données individuelles selon la race au Canada, ce qui représente un obstacle fondamental à l'identification de stratégies de soins visant à améliorer les résultats de santé liés à la COVID-19 pour les populations racialisées et à l'élaboration de politiques publiques visant à mieux soutenir les communautés marginalisées. Des travaux fédéraux, provinciaux et territoriaux sont en cours pour normaliser la déclaration des cas de termes couramment utilisés, comme la race et l'origine ethnique, afin d'éviter une fausse représentation des collectivités et d'obtenir une évaluation adéquate des facteurs de risque associés à la COVID-19 parmi différentes populations. Afin d'améliorer ces méthodes, nous recommandons que les établissements de santé et les chercheurs mobilisent activement les membres et les intervenants de la communauté parmi les groupes démographiques cibles afin d'aider à déterminer les priorités en matière de collecte et d'analyse de données et de production de rapports et d'élaboration et de mise en œuvre de politiques. La collecte de données désagrégées selon la race doit être entreprise avec l'intention expresse de démanteler les schémas de racisme systémique en soins de santé, ce qui ne peut être réalisé qu'en établissant l'équité raciale dans le cycle de vie des données (34).

Notre analyse comporte certaines limites. À ce jour, nous n'avons pas été en mesure d'entreprendre des sous-analyses officielles selon le sexe ou l'âge, compte tenu du manque de données et des diverses façons dont la race est opérationnalisée au Canada et à l'échelle internationale. Nous notons que ces facteurs, ainsi que d'autres, comme le statut socioéconomique, le logement, l'emploi et l'éducation, risquent d'avoir des répercussions différentes sur les populations racialisées et qu'ils nécessitent une étude plus approfondie. Enfin, nous n'avons pas discuté des répercussions de la pandémie de COVID-19 au sein des populations autochtones du Canada, en reconnaissance de la nécessité de faire la distinction entre les identités raciales et autochtones. Les peuples autochtones représentent 4,9\% de la population (35) et ont invariablement eu des résultats de santé plus faibles que ceux des Canadiens non autochtones $(18,36)$. Services aux Autochtones Canada recueille actuellement des données sur le statut d'Autochtone et les résultats associés à la COVID-19.

\section{Conclusion}

Les données désagrégées selon la race sont essentielles à notre compréhension des répercussions de la maladie sur les Canadiens marginalisés, en ce qui concerne la pandémie de la COVID-19 et au-delà. Des preuves croissantes de différences raciales dans les taux d'incidence et de mortalité associés à la COVID-19 ont souligné la nécessité d'améliorer les politiques relatives à la santé des populations racialisées et les interventions ciblées afin d'atténuer les répercussions de la COVID-19. Les efforts visant à recueillir ce type de données au Canada se poursuivent et devraient s'étendre au-delà de la portée de la pandémie afin de cerner les disparités en soins de santé et de trouver des solutions pour réduire cet écart.

\section{Déclaration des auteurs}

E. T. - Méthodologie, recherche, rédaction de la version originale, révision et édition

R. E. - Conceptualisation, méthodologie, recherche, rédaction de la version originale, révision et édition, supervision

N. A. - Rédaction de la version originale, révision et édition

M. S. - Rédaction de la version originale, révision et édition

I. J. - Révision et édition

T. D. - Révision et édition

\section{Intérêts concurrents}

Aucun à déclarer.

\section{Remerciements}

Nous tenons à remercier nos partenaires fédéraux, provinciaux et territoriaux pour le travail en cours dans ce domaine.

\section{Financement}

Aucun à déclarer.

\section{Références}

1. Dong E, Du H, Gardner L. An interactive web-based dashboard to track COVID-19 in real time. Lancet Infect Dis 2020;20(5):533-4. DOI PubMed

2. Agence de la santé publique du Canada. Personnes susceptibles de présenter une forme grave de la maladie ou des complications si elles contractent la COVID-19. Gouvernement du Canada; 2020 (accédé 2020-20-21). https://www.canada.ca/fr/sante-publique/services/ publications/maladies-et-affections/personnes-susceptiblesgravement-malades-contractent-covid-19.html 
3. Li J, Huang DQ, Zou B, Yang H, Hui WZ, Rui F, Yee NT, Liu C, Nerurkar SN, Kai JC, Teng ML, Li X, Zeng H, Borghi JA, Henry L, Cheung R, Nguyen MH. Epidemiology of COVID-19: A systematic review and meta-analysis of clinical characteristics, risk factors, and outcomes. J Med Virol 2021;93(3):1449-58. DOI PubMed

4. Institut canadien d'information sur la santé. Normes proposées pour les données fondées sur la race et l'identité autochtone. Ottawa (ON) : ICIS; 2020. https://www.cihi.ca/ $\mathrm{fr} /$ normes-proposees-pour-les-donnees-fondees-sur-la-raceet-lidentite-autochtone

5. National Collaborating Centre for Determinants of Health. Let's Talk: Racism and Health Equity. Antigonish (NS): NCCDH; 2018. https://nccdh.ca/resources/entry/lets-talkracism-and-health-equity

6. Nestel S. Colour Coded Health Care: The Impact of Race and Racism on Canadians' Health. Toronto (ON): Wellesley Institute; 2012. https://www.wellesleyinstitute.com/wpcontent/uploads/2012/02/Colour-Coded-Health-Care.pdf

7. Agence de la santé publique du Canada. Déterminants sociaux et iniquités en santé des Canadiens Noirs : un aperçu. Ottawa (ON) : ASPC; 2020. https://www.canada. $\mathrm{ca} / \mathrm{fr} /$ sante-publique/services/promotion-sante/santepopulation/est-determine-sante/determinants-sociauxiniquites-canadiens-noirs-apercu.html

8. Henry F, Tator C. The Colour of Democracy: Racism in Canadian Society. $4^{\text {th }}$ edition. Toronto: Nelson Education; 2010.

9. Paradies $Y$, Ben J, Denson N, Elias A, Priest N, Pieterse A, Gupta A, Kelaher M, Gee G. Racism as a Determinant of Health: A Systematic Review and Meta-Analysis. PLoS One 2015;10(9):e0138511. DOI PubMed

10. Centers for Disease Control and Prevention. Health Equity Considerations and Racial and Ethnic Minority Groups. Atlanta (GA): CDC; 2020. https://www.cdc.gov/ coronavirus/2019-ncov/community/health-equity/raceethnicity.html

11. Feagin J, Bennefield Z. Systemic racism and U.S. health care. Soc Sci Med 2014;103:7-14. DOl PubMed

12. Yancy CW. COVID-19 and African Americans. JAMA 2020;323(19):1891-2. DOI PubMed

13. Williamson EJ, Walker AJ, Bhaskaran K, Bacon S, Bates C, Morton CE, Curtis HJ, Mehrkar A, Evans D, Inglesby P, Cockburn J, McDonald HI, MacKenna B, Tomlinson L, Douglas IJ, Rentsch CT, Mathur R, Wong AY, Grieve R, Harrison D, Forbes H, Schultze A, Croker R, Parry J, Hester F, Harper S, Perera R, Evans SJ, Smeeth L, Goldacre B. Factors associated with COVID-19-related death using OpenSAFELY. Nature 2020;584(7821):430-6. DOI PubMed

14. Deaton Review IF. Are some ethnic groups more vulnerable to COVID-19 than others? (accédé 2020-10-18). https://ifs. org.uk/inequality/chapter/are-some-ethnic-groups-morevulnerable-to-covid-19-than-others/
15. The COVID Tracking Project. The COVID Racial Data Tracker (accédé 2020-10-23). https://covidtracking.com/race

16. Ottawa Neighbourhood Study. COVID-19 in Ottawa Neighbourhoods (accédé 2020-10-20). https:// www.neighbourhoodstudy.ca/covid-19-in-ottawaneighbourhoods/

17. Ahmad K, Erqou S, Shah N, Nazir U, Morrison AR, Choudhary G, Wu WC. Association of poor housing conditions with COVID-19 incidence and mortality across US counties. PLoS One 2020;15(11):e0241327. DOI PubMed

18. Agence de la santé publique du Canada. Les principales inégalités en santé au Canada - Sommaire exécutif. Ottawa (ON) : ASPC; 2018 (accédé 2020-12-04). https://www. canada.ca/fr/sante-publique/services/publications/sciencerecherche-et-donnees/rapport-principales-inegalites-santecanada-sommaire-executif.html

19. Agence ontarienne de protection et de promotion de la santé (Santé publique Ontario). La COVID-19 en Ontario un regard sur la diversité : du 15 janvier 2020 au 14 mai 2020. Toronto, ON. Imprimeur de la Reine pour I'Ontario, 2020. https://www.publichealthontario.ca/-/media/ documents/ncov/epi/2020/06/covid-19-epi-diversity. $\mathrm{pdf} ? \mathrm{la}=\mathrm{fr}$

20. Chung $H$, Fung $K$, Ferreira-Legere LE, Chen $B$, Ishiguro $L$, Kalappa G, Gozdyra P, Campbell T, Paterson JM, Bronskill SE, Kwong JC, Guttmann A, Azimaee M, Vermeulen MJ, Schull MJ. COVID-19 Laboratory Testing in Ontario: Patterns of Testing and Characteristics of Individuals Tested, as of April 30, 2020. Toronto (ON): ICES; 2020. https://www.ices.on.ca/Publications/Atlases-andReports/2020/COVID-19-Laboratory-Testing-in-Ontario

21. Whittle RS, Diaz-Artiles A. An ecological study of socioeconomic predictors in detection of COVID-19 cases across neighborhoods in New York City. BMC Med 2020;18(1):271. DOI PubMed

22. Sze $S$, Pan D, Nevill CR, Gray LJ, Martin CA, Nazareth J, Minhas JS, Divall P, Khunti K, Abrams KR, Nellums LB, Pareek M. Ethnicity and clinical outcomes in COVID-19: A systematic review and meta-analysis. EClinicalMedicine 2020;29:100630. DOI PubMed

23. Martin Turcotte, Katherine Savage. La contribution des immigrants et des groupes de population désignés comme minorités visibles aux professions d'aide-infirmier, d'aide-soignant et de préposé aux bénéficiaires. Ottawa (ON) : Statistique Canada; juin 2020. https://www150. statcan.gc.ca/n1/pub/45-28-0001/2020001/article/00036-fra. htm

24. Kirby T. Evidence mounts on the disproportionate effect of COVID-19 on ethnic minorities. Lancet Respir Med 2020;8(6):547-8. DOI PubMed

25. Hutchins SS, Fiscella K, Levine RS, Ompad DC, McDonald M. Protection of racial/ethnic minority populations during an influenza pandemic. Am J Public Health 2009;99 Suppl 2:S261-70. DOI PubMed 
26. Thomson EM, Kalayci H, Walker M. Conséquences cumulatives de l'exposition à des stresseurs chez les Canadiens : un profil de la charge allostatique. Statistique Canada; Rapport sur la santé 2019;30(6):14-21.

https://www.doi.org/10.25318/82-003-x201900600002-fra

27. McEwen BS, Stellar E. Stress and the individual. Mechanisms leading to disease. Arch Intern Med 1993;153(18):2093-101. DOI PubMed

28. Subedi R, Greenberg L, Turcotte M. Taux de mortalité attribuable à la COVID-19 dans les quartiers ethnoculturels du Canada. Ottawa (ON) : Statistique Canada; (modifié 2020-10). https://www150.statcan.gc.ca/n1/pub/4528-0001/2020001/article/00079-fra.htm

29. Bray I, Gibson A, White J. Coronavirus disease 2019 mortality: a multivariate ecological analysis in relation to ethnicity, population density, obesity, deprivation and pollution. Public Health 2020;185:261-3. DOI PubMed

30. Public Health Agency of Canada. Emerging Sciences Group. Emerging Evidence on COVID-19: Evidence Brief on Ethnicity and COVID-19. Ottawa (ON): PHAC; Sep 2020. Full report available from: phac. ocsoevidence-bcscdonneesprobantes.aspc@canada.ca
31. Agency for Healthcare Research and Quality. Improving Data Collection across the Health Care System. Rockville, MD: Agency for Healthcare Research and Quality (accédé 2020-12-07). https://www.ahrq.gov/ research/findings/final-reports/iomracereport/reldata5.html

32. Browne AJ, Varcoe CM, Wong ST, Smye VL, Khan KB. Can ethnicity data collected at an organizational level be useful in addressing health and healthcare inequities? Ethn Health 2014;19(2):240-54. DOI PubMed

33. Varcoe C, Browne AJ, Wong S, Smye VL. Harms and benefits: collecting ethnicity data in a clinical context. Soc Sci Med 2009;68(9):1659-66. DOI PubMed

34. Nelson AL, Zanti S. A framework for centering racial equity throughout the administrative data life cycle. Int J Popul Data Sci 2020;5(1):1367. DOI PubMed

35. Statistique Canada. Statistiques sur les Peuples autochtones. Ottawa (ON) : Statistique Canada; 2019 (accédé 2020-12-17). https://www.statcan.gc.ca/fra/sujetsdebut/peuples_autochtones

36. Greenwood ML, de Leeuw SN. Social determinants of health and the future well-being of Aboriginal children in Canada. Paediatr Child Health 2012;17(7):381-4. PubMed 